\title{
Pro-oxidant activity of sulforaphane and cisplatin potentiates apoptosis and simultaneously promotes autophagy in malignant mesothelioma cells
}

\author{
YOON-JIN LEE and SANG-HAN LEE \\ Department of Biochemistry, College of Medicine, Soonchunhyang University, Cheonan 330-930, Republic of Korea
}

Received July 1, 2016; Accepted March 6, 2017

DOI: $10.3892 / \mathrm{mmr} .2017 .6789$

\begin{abstract}
Sulforaphane (SFN) is an isothiocyanate compound derived from glucoraphanin, which is found in cruciferous vegetables, and has been heralded as a chemopreventive and/or chemotherapeutic agent. The present study investigated the effects of SFN on enhancing the anticancer role of cisplatin (cis-dichlorodiammineplatinum; CDDP) in H-28 malignant mesothelioma cells. At concentrations demonstrating limited toxicity in MeT-5A normal human mesothelial cells, combination treatment with the two compounds exhibited synergistic growth-inhibiting and apoptosis-promoting activities, as demonstrated by a series of proapoptotic events, including reactive oxygen species accumulation, loss of mitochondrial membrane potential, upregulation of p53 expression, increased B-cell lymphoma 2 (Bcl-2) associated $\mathrm{X}$ protein/Bcl-2 ratio, activation of caspase-3, the occurrence of a sub- $G_{0} / G_{1}$ peak and an increase in cells with pyknotic and fragmented nuclei, Annexin V-phycoerythrin-positive staining and $\mathrm{G}_{2} / \mathrm{M}$ phase-transition delay in the cell cycle. The phosphorylation levels of Akt and mammalian target of rapamycin were reduced by the combination treatment, which was accompanied by a significant increase in the level of autophagosomal marker protein microtubule-associated protein 1 light chain 3B-II and the accumulation of acidic vesicular organelles. Pretreatment with the antioxidant N-acetylcysteine attenuated both apoptosis and autophagy, whereas inhibition of autophagy by bafilomycin A1 potentiated apoptotic cell death following the combination treatment with SFN and CDDP. Considering the pro-oxidant-based combinational approach, the results of the present study provide a rationale for targeting cytoprotective autophagy as a potential therapeutic strategy for malignant mesothelioma.
\end{abstract}

Correspondence to: Professor Sang-Han Lee, Department of Biochemistry, College of Medicine, Soonchunhyang University, 31 Soonchunhyang 6-gil, Dongnam-gu, Cheonan 330-930, Republic of Korea

E-mail: m1037624@sch.ac.kr

Key words: mesothelioma, sulforaphane, cisplatin, reactive oxygen species, apoptosis, autophagy

\section{Introduction}

Malignant mesothelioma (MM) is a rare and aggressive tumor; its occurrence is associated with substantial exposure to airborne asbestos fibers (1). Approximately $70 \%$ of the reported cases of MM occur in the pleura, with a further $\sim 30 \%$ occurring in the peritoneum and $1-2 \%$ in the pericardium and the tunica vaginalis testis (2). The incidence of $\mathrm{MM}$ is increasing worldwide owing to an increase in asbestos exposure earlier in life; however, owing to the long latency period, MM is often diagnosed late in life (3). MM is mostly treated with chemotherapy over the course of the disease (3).

Platinum compounds, such as cisplatin (cis-dichlorodiammineplatinum; CDDP), are currently regarded as first-line treatments for patients with MM, although their efficacy remains limited (4). These drugs inhibit DNA synthesis through the formation of intrastrand cross-links and the formation of DNA adducts. A majority of patients eventually relapse with the emergence of CDDP-resistant cell populations, despite the initial response to therapy. Previously published results from a phase III trial in patients with MM indicated that the combination therapy of CDDP with pemetrexed improved response rates (41.3 vs. 16.7\%), median survival time (12.1 vs. 9.3 months) and median time to progressive disease (5.7 vs. 3.9 months) compared with CDDP alone (5). A number of strategies, including various drug combinations, have been used to prevent or delay resistance to chemotherapeutic agents; however, it currently appears inevitable that drug-resistant variants will emerge during disease progression. It has been suggested that functional defects in apoptosis signaling are important for the development of chemoresistance in MM (6). Therefore, the development of effective chemosensitizing agents that represent distinct and complementary modes of killing cells may have important clinical implications. Sulforaphane (SFN) is an isothiocyanate compound derived from glucoraphanin, which is present in cruciferous vegetables, and has previously been demonstrated to have the ability to suppress malignant cancer phenotypes, including cell proliferation, angiogenesis and metastasis (7). SFN has also been reported to sensitize cancer cells to imatinib- and tumor necrosis factor-related apoptosis inducing ligand-induced apoptosis, via a reactive oxygen species (ROS)-dependent pathway $(8,9)$, suggesting potential therapeutic value as an adjunct to current cancer therapies. 
Autophagy is an evolutionarily conserved catabolic process in which damaged or dysfunctional cellular components are fused with a lysosome for degradation and recycled for use in the metabolic and biosynthetic pathways. Autophagy is considered an adaptive response that promotes survival against cellular stresses; in other cases, however, prolonged activation of autophagy appears to promote cell death, termed autophagic cell death (10). As a way to deal with cellular stresses, such as glucose deprivation, hypoxia, oxidative stress and endoplasmic reticulum stress, cancer cells utilize the autophagic pathway, which enables continued growth by maintaining cellular energy production (11). Previous research has indicated that the inhibition of autophagy may potentiate cancer cell death and enhance the efficacy of anticancer drugs on cells that use autophagy to survive $(12,13)$.

The present study aimed to identify the chemosensitizing effect of SFN and to understand the implication of autophagy in MM cell resistance to CDDP therapy. The effects of SFN on enhancing the anticancer role of CDDP in H-28 MM cells were evaluated, as well as alterations to apoptosis and autophagy. Potential roles of autophagy as a factor for MM cell survival are discussed.

\section{Materials and methods}

Reagents and cell culture. SFN, CDDP, 3-(4,5-dimethylthiazol-2-yl)-2,5-diphenyltetrazolium bromide (MTT), Rhodamine 123, bafilomycin A1, N-acetylcysteine (NAC), propidium iodide (PI), DAPI, 2',7'-dichlorodihydrofluorescein diacetate (DCF-DA) and the $\beta$-actin antibody (catalog no. A2228) were obtained from Sigma-Aldrich (Merck KGaA, Darmstadt, Germany). Antibodies against B-cell lymphoma 2 (Bcl-2; catalog no. 2820), Bcl-2 associated X (Bax; catalog no. 5023), cyclin D1 (catalog no. 2978), cyclin B1 (catalog no. 12231), phosphorylated (p)-cyclin dependent kinase 2 (p-Cdc2 ${ }^{\text {Tyr15}}$; catalog no. 4539), Akt (catalog no. 9272), p-Akt (catalog no. 9271), mTOR (catalog no. 2972), p-mTOR (catalog no. 2971), microtubule-associated protein 1 light chain $3 \mathrm{~B}$ (LC3B; catalog no. 3868), PARP (catalog no. 9542), cleaved PARP (catalog no. 9541), procaspase-3 (catalog no. 9665), and cleaved caspase-3 (catalog no. 9664) were purchased from Cell Signaling Technology, Inc. (Danvers, MA, USA). Antibodies against p53 (catalog no. sc-126), p21 WAF1/CIP1 (catalog no. sc-6246), goat anti-rabbit IgG-HRP (catalog no. sc-2004), goat anti-mouse IgG-HRP (catalog no. sc-2005), and the Enhanced Chemiluminescence (ECL) kit were purchased from Santa Cruz Biotechnology, Inc. (Dallas, TX, USA).

The human malignant mesothelioma cell line $\mathrm{H}-28$ and the human mesothelial cell line MeT-5A were obtained from the American Type Culture Collection (ATCC; Manassas, VA, USA). H-28 cells were maintained at $37^{\circ} \mathrm{C}$ in RPMI-1640 medium (catalog no. SH30027.01; GE Healthcare Life Sciences, Chalfont, UK) supplemented with $10 \%$ fetal bovine serum (FBS; catalog no. SH30084.03; GE Healthcare Life Sciences), $100 \mathrm{U} / \mathrm{ml}$ penicillin and $100 \mu \mathrm{g} / \mathrm{ml}$ streptomycin. MeT-5A cells were maintained in M-199 medium (Welgene, Inc., Daegu, Korea) supplemented with $3.3 \mathrm{nM}$ epidermal growth factor (catalog no. E9644; Sigma-Aldrich), 5\% FBS,
$100 \mathrm{U} / \mathrm{ml}$ penicillin and $100 \mu \mathrm{g} / \mathrm{ml}$ streptomycin. Cells were grown to $70 \%$ confluence in a submerged monolayer culture for $24 \mathrm{~h}$ prior to treatment.

Cell viability assay. Cell viability was measured using the MTT assay. Cells (5x103 cells/well) were seeded into 96-well microtiter plates and incubated with the vehicle $[0.1 \%$ dimethylsulfoxide (DMSO) in medium], SFN (0, 10, 20, 30, 40, 50, 60, 80 and $100 \mu \mathrm{M}), \operatorname{CDDP}(0,40,80,160,240,480,640$ and $960 \mu \mathrm{M}$ ), or a combination of SFN and CDDP (20 and $40 \mu \mathrm{M}$, respectively) for $48 \mathrm{~h}$ at $37^{\circ} \mathrm{C}$, and then exposed to MTT (final concentration, $0.1 \mathrm{mg} / \mathrm{ml}$ ) for an additional $4 \mathrm{~h}$ at $37^{\circ} \mathrm{C}$. Absorbance values were measured at a wavelength of $560 \mathrm{~nm}$ with a GloMax-Multi Microplate Multimode Reader (Promega Corporation, Madison, WI, USA). The number of viable cells was determined by comparing results with the vehicle-treated control cells. The two-compound combination effect was evaluated by calculating the combination index (CI), as previously described (14); where $\mathrm{CI}<1$ indicates a synergistic effect, $\mathrm{CI}=1$ indicates an additive effect and $\mathrm{CI}>1$ indicates an antagonistic effect.

DAPI staining. Nuclear condensation and fragmentation were observed by DAPI staining. Cells $\left(1 \times 10^{5}\right.$ cells/well) were seeded into a 6-well culture plate and incubated with SFN $(20 \mu \mathrm{M})$ and CDDP $(40 \mu \mathrm{M})$, alone or in combination, for $48 \mathrm{~h}$ at $37^{\circ} \mathrm{C}$. The cells were trypsinized, pelleted by centrifugation at $500 \mathrm{x} \mathrm{g}$ for $7 \mathrm{~min}$ at $4^{\circ} \mathrm{C}$, and fixed in $100 \%$ methanol at room temperature for $20 \mathrm{~min}$. After centrifugation, the pellet was resuspended in DAPI $(2 \mu \mathrm{g} / \mathrm{ml})$ for $10 \mathrm{~min}$ in the dark and washed with $1 \mathrm{X}$ PBS. Cells were spread on slides and the coverslip was then mounted using mounting medium (catalog no. 08381; Polysciences, Inc., Warrington, PA, USA). Apoptotic cells were observed with a FluoView FV10i confocal fluorescence microscope (Olympus Corporation, Tokyo, Japan). Five random fields were analyzed on each slide.

Cell cycle analysis. Cell cycle distribution was measured by quantitation of DNA content in the PI-stained cells. Trypsinized cells $\left(\sim 10^{6}\right.$ cell $\left./ \mathrm{ml}\right)$ were pelleted by centrifugation at $500 \mathrm{x} \mathrm{g}$ for $7 \mathrm{~min}$ at $4^{\circ} \mathrm{C}$, fixed in $70 \%$ ice-cold ethanol overnight at $-20^{\circ} \mathrm{C}$ and treated with DNase-free RNase A $(150 \mu \mathrm{g} / \mathrm{ml})$ and PI $(20 \mu \mathrm{g} / \mathrm{ml})$ for $1 \mathrm{~h}$ at $4^{\circ} \mathrm{C}$. Data from 10,000 single-cell events were collected by a MACSQuant Analyzer flow cytometer and analyzed using MACSQuantify ${ }^{\mathrm{TM}}$ software version 2.5 (Miltenyi Biotec GmbH, Bergisch Gladbach, Germany).

Apoptosis assay. Apoptotic cell distribution was determined with a Muse Annexin V and Dead Cell Assay kit (catalog no. MCH100105; Merck KGaA, Darmstadt, Germany), according to the manufacturer's protocol. This kit includes a fluorescent dye phycoerythrin (PE) conjugated to Annexin V to detect phosphatidylserine on the external membrane of apoptotic cells and 7-amino-actinomycin D as a dead cell marker. Briefly, cells $\left(1 \times 10^{5}\right.$ cells/well) were seeded into a 6-well culture plate and incubated with SFN $(20 \mu \mathrm{M})$ and CDDP $(40 \mu \mathrm{M})$, alone or in combination, for $48 \mathrm{~h}$ at $37^{\circ} \mathrm{C}$. The cells were trypsinized and collected into culture medium, mixed with the Muse Annexin V \& Dead Cell reagent, and analyzed using a Muse Cell Analyzer (Merck KGaA). 
Western blot analysis. A total of $1 \times 10^{5}$ cells/well were seeded into 6-well culture plate and incubated with or without NAC $(5 \mathrm{mM})$ or bafilomycin $\mathrm{A} 1(50 \mathrm{nM})$ for $2 \mathrm{~h}$ at $37^{\circ} \mathrm{C}$ prior to co-treatment with SFN $(20 \mu \mathrm{M})$ and CDDP $(40 \mu \mathrm{M})$ for a further $48 \mathrm{~h}$. Cells were lysed in radioimmunoprecipitation assay buffer [1X PBS, 1\% NP-40, 0.5\% sodium deoxycholate, $0.1 \%$ SDS, $10 \mu \mathrm{g} / \mathrm{ml}$ phenylmethanesulfonylfluoride and a protease inhibitor cocktail tablet (Boehringer Mannheim, Germany)] for $30 \mathrm{~min}$ on ice, and subsequently pelleted by centrifugation $10,000 \mathrm{x}$ g for $10 \mathrm{~min}$ at $4{ }^{\circ} \mathrm{C}$. The protein concentration was determined using a bicinchoninic acid assay kit (catalog no. 23225; Pierce $^{\mathrm{TM}}$; Thermo Scientific, Rockford, IL, USA), according to the manufacturer's protocol. Cell lysates containing $40 \mu \mathrm{g}$ protein were separated on NuPAGE 4-12\% Bis-Tris polyacrylamide gels (Invitrogen; Thermo Fisher Scientific, Inc., Waltham, MA, USA) and electrophoretically transferred to Immuno-Blot polyvinylidene fluoride membranes (Bio-Rad Laboratories, Inc., Hercules, CA, USA). The membrane was incubated overnight at $4^{\circ} \mathrm{C}$ with primary antibodies diluted to 1:500 in casein blocking buffer (catalog no. B6429; Sigma-Aldrich), followed by incubation for $2 \mathrm{~h}$ at room temperature with a secondary antibody coupled to horseradish peroxidase diluted 1:5,000 in casein blocking buffer. Signals were visualized using an ECL detection kit and X-ray film. Blots were stripped using a stripping buffer $(100 \mathrm{mM}$ $\beta$-mercaptoethanol, 2\% SDS, and $62.5 \mathrm{mM}$ Tris- $\mathrm{HCl}, \mathrm{pH} 6.7$ ) and re-probed with an anti- $\beta$-actin antibody to serve as the loading control.

Measurement of intracellular ROS levels. Intracellular ROS levels were evaluated by measuring DCF-DA (Sigma-Aldrich) fluorescence intensity. Briefly, cells $\left(1 \times 10^{5}\right.$ cells/well) were seeded into a 6-well culture plate and incubated with or without $5 \mathrm{mM} \mathrm{NAC}$ for $2 \mathrm{~h}$ at $37^{\circ} \mathrm{C}$ prior to co-treatment with $20 \mu \mathrm{M}$ SFN and $40 \mu \mathrm{M}$ CDDP for a further $48 \mathrm{~h}$. The cells were trypsinized, pelleted by centrifugation at $500 \mathrm{x} \mathrm{g}$ for $7 \mathrm{~min}$ at $4^{\circ} \mathrm{C}$, and resuspended in serum-free RPMI-1640 medium containing $10 \mu \mathrm{M}$ DCF-DA for $30 \mathrm{~min}$ at $37^{\circ} \mathrm{C}$ in the dark. Following incubation, cells were washed twice with $1 \mathrm{X}$ PBS, trypsinized, resuspended in $1 \mathrm{X}$ PBS, and immediately analyzed with a MACSQuant Analyzer flow cytometer and MACSQuantify $^{\mathrm{TM}}$ software version 2.5 (Miltenyi Biotec $\mathrm{GmbH})$. DCF fluorescence was detected using a $530 \mathrm{~nm}$ bandpass filter, and each measurement was based on the mean fluorescence intensity of 10,000 cells.

Mitochondrial membrane potential $(\Delta \Psi m)$ analysis. Cells $\left(5 \times 10^{4}\right.$ cells/well) were seeded onto 6 -well plates and incubated with or without NAC $(5 \mathrm{mM})$ for $2 \mathrm{~h}$ at $37^{\circ} \mathrm{C}$ prior to co-treatment with $20 \mu \mathrm{M} \mathrm{SFN}$ and $40 \mu \mathrm{M}$ CDDP for a further $48 \mathrm{~h}$. The cells were trypsinized, harvested by centrifugation at $500 \mathrm{x} \mathrm{g}$ for $7 \mathrm{~min}$ at $4^{\circ} \mathrm{C}$, washed twice with PBS, and stained with serum-free RPMI-1640 medium containing Rhodamine 123 (final concentration, $30 \mathrm{nM}$ ) at $37^{\circ} \mathrm{C}$ for $30 \mathrm{~min}$. Following incubation, cells were washed twice with $1 \mathrm{X}$ PBS, trypsinized and resuspended in $1 \mathrm{X}$ PBS. Fluorescence intensity was measured and analyzed using a MACSQuant analyzer flow cytometer and MACSQuantify ${ }^{\mathrm{TM}}$ software version 2.5 (Miltenyi Biotec $\mathrm{GmbH})$.
Measurement of reduced glutathione (GSH)/oxidized glutathione (GSSG) ratio. Total glutathione (that is, total combined GSH and GSSG expression) and GSSG levels were measured using a GSH/GSSG-Glo assay (Promega Corporation), according to the manufacturer's protocol. Briefly, cells $\left(5 \times 10^{3}\right.$ cells/well $)$ were seeded into a 96 -well culture plate and treated with the vehicle (0.1\% DMSO in RMPI-1640 medium) or NAC $(5 \mathrm{mM})$ for $2 \mathrm{~h}$ at $37^{\circ} \mathrm{C}$ prior to co-treatment with $20 \mu \mathrm{M}$ SFN and $40 \mu \mathrm{M}$ CDDP for a further $48 \mathrm{~h}$. The media was subsequently removed and replaced with total glutathione or GSSG lysis reagent, for total glutathione or GSSG measurement, respectively. The GSH probe, luciferin-NT, was converted to luciferin, which is coupled to a firefly luciferase. The GSH/GSSG ratio was calculated from the luminescence measurements using a GloMax-Multi Microplate Multimode Reader (Promega Corporation). The GSSG value was subtracted from the total glutathione to calculate the level of GSH.

Flow cytometry analysis of lysosomes. Cells (1x10 5 cells/well) were seeded into a 6-well culture plate and incubated with or without $5 \mathrm{mM} \mathrm{NAC}$ for $2 \mathrm{~h}$ at $37^{\circ} \mathrm{C}$ prior to co-treatment with $20 \mu \mathrm{M}$ SFN and $40 \mu \mathrm{M}$ CDDP for a further $48 \mathrm{~h}$. Changes in the activation of the autophagosome/lysosome pathway were quantitatively determined by staining the acidic vacuoles inside the cells with LysoTracker Red DND-99 (25 nmol/l; Molecular Probes; Thermo Fisher Scientific, Inc.) for $20 \mathrm{~min}$ at $37^{\circ} \mathrm{C}$. Following incubation, cells were washed twice with $1 \mathrm{X}$ PBS, trypsinized and resuspeded in $1 \mathrm{X}$ PBS. The fluorescence intensity of the cells was analyzed on channel APC-A using a MACSQuant analyzer flow cytometer and MACSQuantify ${ }^{\mathrm{TM}}$ software version 2.5 (Miltenyi Biotec $\mathrm{GmbH}$ ).

Statistical analysis. Statistical comparisons were performed by one-way analysis of variance followed by Tukey's post hoc correction for multiple comparisons, using SPSS version 17.0 (SPSS, Inc., Chicago, IL, USA). Data were expressed as the mean \pm standard deviation. $\mathrm{P}<0.05$ was considered to indicate a statistically significant difference.

\section{Results}

Synergistic growth-inhibiting and apoptosis-promoting effects of combination SFN/CDDP treatment. The cytotoxic effects of SFN and CDDP exposure were assessed by treating H-28 MM cells and MeT-5A normal human mesothelial cells with increasing concentrations of SFN (10-100 $\mu \mathrm{M})$ or CDDP (40-960 $\mu \mathrm{M})$ for $48 \mathrm{~h}$ and analyzed by MTT assay. As demonstrated in Fig. 1A, treatment with SFN or CDDP alone inhibited the growth of MeT-5A and $\mathrm{H}-28$ cells in a concentration-dependent manner. MeT-5A cells exhibited lower cytotoxicity to the two compounds when compared with $\mathrm{H}-28$ cells. Concentrations of 20 and $40 \mu \mathrm{M}$ for the combination of SFN and CDDP, respectively, were chosen based on the concentrations showing a high cell viability (>90\%) in MeT-5A cells when treated with SFN (92\%) or CDDP (95\%) alone. Combination treatment of $20 \mu \mathrm{M}$ SFN and $40 \mu \mathrm{M}$ CDDP (SFN/CDDP) led to a significant decrease in the viability $(62.5 \%)$ of $\mathrm{H}-28$ cells compared with $20 \mu \mathrm{M} \mathrm{SFN}$ (85.3\%) or $40 \mu \mathrm{M}$ CDDP $(96.7 \%)$ treatment alone. However, 
A

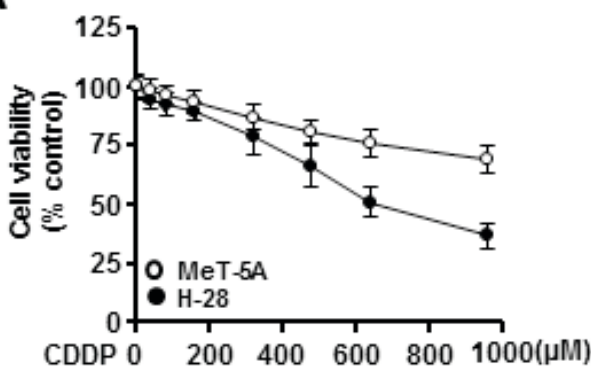

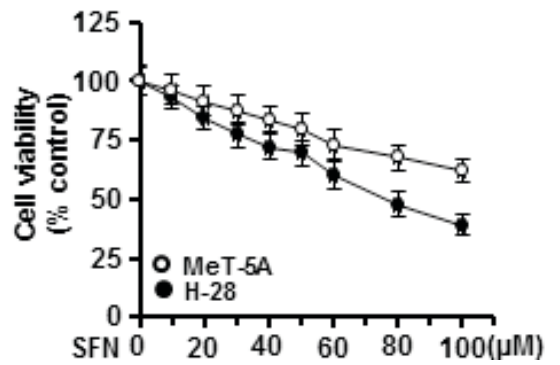

B

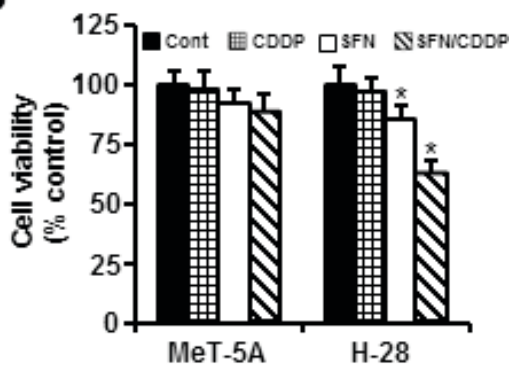

Figure 1. Synergistic cytotoxic effects of SFN and CDDP treatment in mesothelial cells. (A) H-28 malignant mesothelioma cells and MeT-5A normal human mesothelial cells were treated with the vehicle $(0.1 \%$ DMSO) or various concentrations of SFN (10-100 $\mu \mathrm{M})$ or CDDP $(40-960 \mu \mathrm{M})$ for $48 \mathrm{~h}$. (B) Cells were treated with SFN $(20 \mu \mathrm{M})$ and CDDP $(40 \mu \mathrm{M})$, alone or in combination, for $48 \mathrm{~h}$. Cell viability was determined using an MTT assay. Error bars indicate the mean \pm standard deviation for three independent experiments. " $\mathrm{P}<0.05$ vs. respective controls. CDDP, cis-dichlorodiammineplatinum (cisplatin); Cont, DMSO-treated control; SFN, sulforaphane.

A
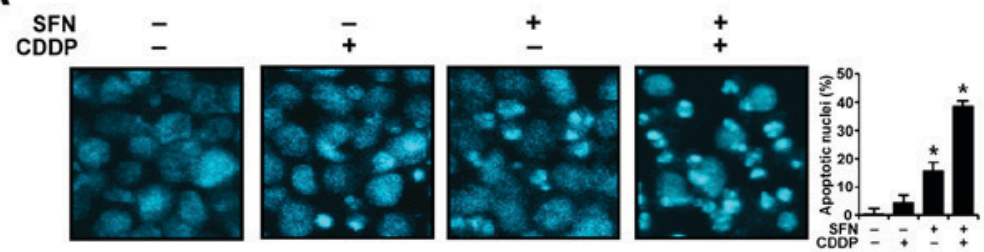

B

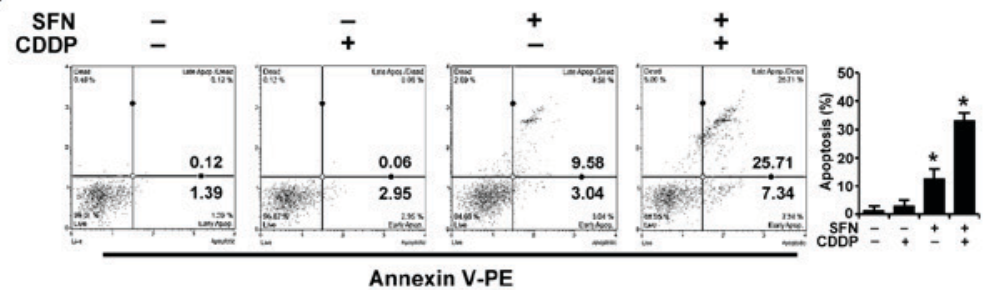

C

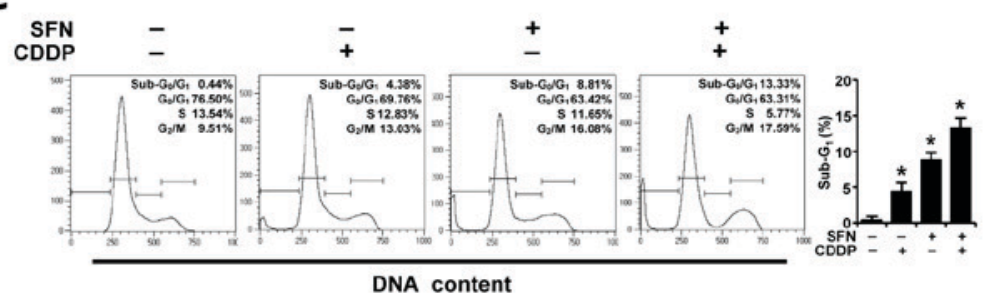

D
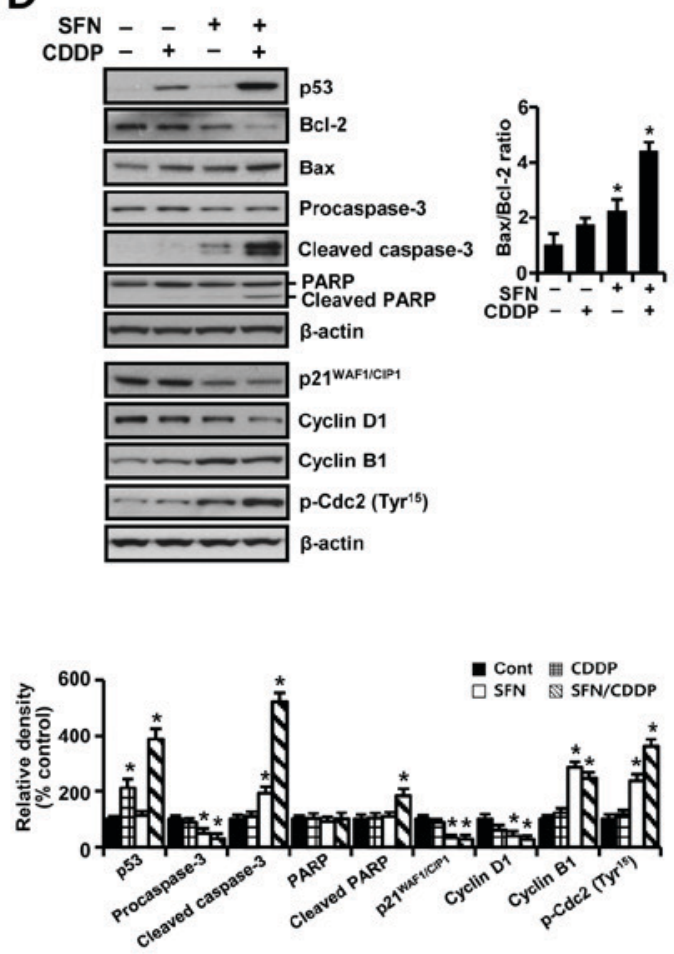

Figure 2. Apoptosis-promoting and growth-inhibiting effects of SFN and CDDP in H-28 malignant mesothelioma cells. H-28 cells were treated with SFN $(20 \mu \mathrm{M})$ and CDDP $(40 \mu \mathrm{M})$, alone or in combination, for $48 \mathrm{~h}$. (A) Nuclear morphology was assessed by nuclear staining with DAPI (magnification $\mathrm{x} 40$ ). (B) The number of apoptotic cells following Annexin V-PE staining was analyzed using a Muse Cell Analyzer. (C) Cell distribution in sub- $\mathrm{G}_{0} / \mathrm{G}_{1}, \mathrm{G}_{0} / \mathrm{G}_{1}, \mathrm{~S}$ and $\mathrm{G}_{2} / \mathrm{M}$ phases was analyzed by flow cytometry following staining with propidium iodide $(20 \mu \mathrm{g} / \mathrm{ml})$. (D) Expression levels of apoptosis- and cell cycle-regulating proteins were measured by western blot analysis. The Bax/Bcl-2 ratio and relative density of protein bands were obtained from densitometric analysis of the western blot images normalized to $\beta$-actin. Representative results are presented from one of three independent experiments; $\beta$-actin was used as a loading control. Error bars indicate the mean \pm standard deviation for three independent experiments. ${ }^{*} \mathrm{P}<0.05$ vs. respective untreated controls. Bax, Bcl-2 associated X protein; Bcl-2, B-cell lymphoma 2; CDDP, cis-dichlorodiammineplatinum (cisplatin); PARP, poly(ADP-ribose) polymerase; p-Cdc2; phosphorylated cyclin dependent kinase 2; PE, phycoerythrin; SFN, sulforaphane.

the combination of $20 \mu \mathrm{M} \mathrm{SFN}$ and $40 \mu \mathrm{M}$ CDDP did not significantly inhibit the viability (88.3\%) of MeT-5A cells compared with $20 \mu \mathrm{M}$ SFN (91.6\%) or $40 \mu \mathrm{M}$ CDDP (98.5\%) alone (Fig. 1B). Based on the CI value, the cytotoxic effect of SFN and CDDP in combination on H-28 cells was synergistic (CI=0.454).

To further elucidate whether the growth inhibition of $\mathrm{H}-28$ cells by SFN/CDDP treatment was related to apoptotic cell death, the proapoptotic effects of the two compounds was examined by analyzing nuclear phenotypes and apoptotic cells using DAPI and Annexin V-phycoerythrin (PE) staining, respectively. The proportion of adherent cells with pyknotic and fragmented nuclei increased (Fig. 2A), and the proportion of Annexin V-PE-positive cells undergoing apoptosis increased to $33.05 \%$ (Fig. 2B) in combination treated cells relative to SFN $(12.62 \%)$ or CDDP $(3.01 \%)$ treatment alone. In addition, cell cycle analysis in the PI-stained cells indicated an increase in the sub- $\mathrm{G}_{0} / \mathrm{G}_{1}$ peak, which was indicative of apoptosis (Fig. 2C). 
A
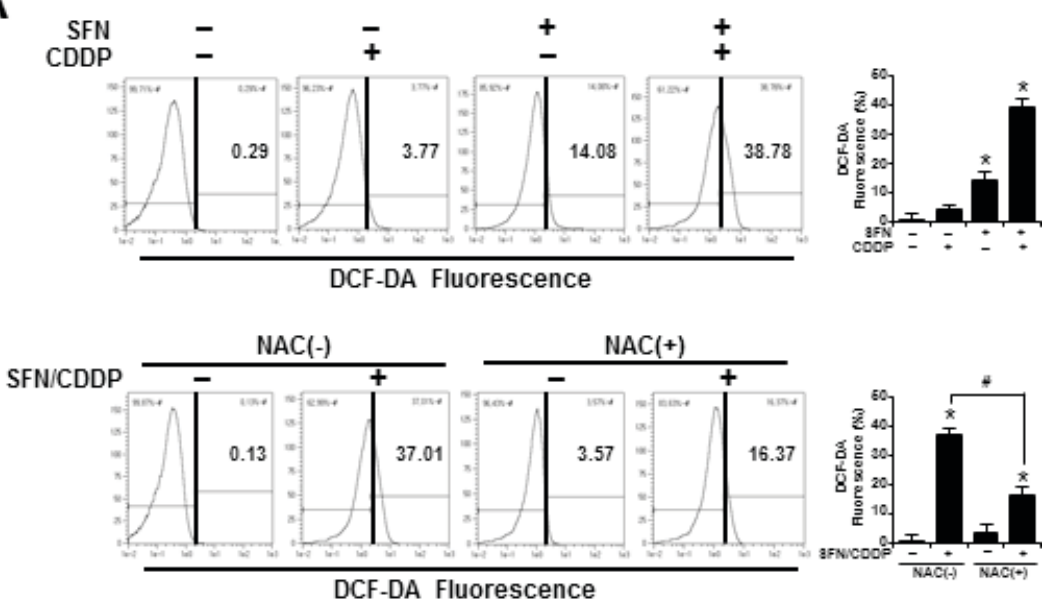

B
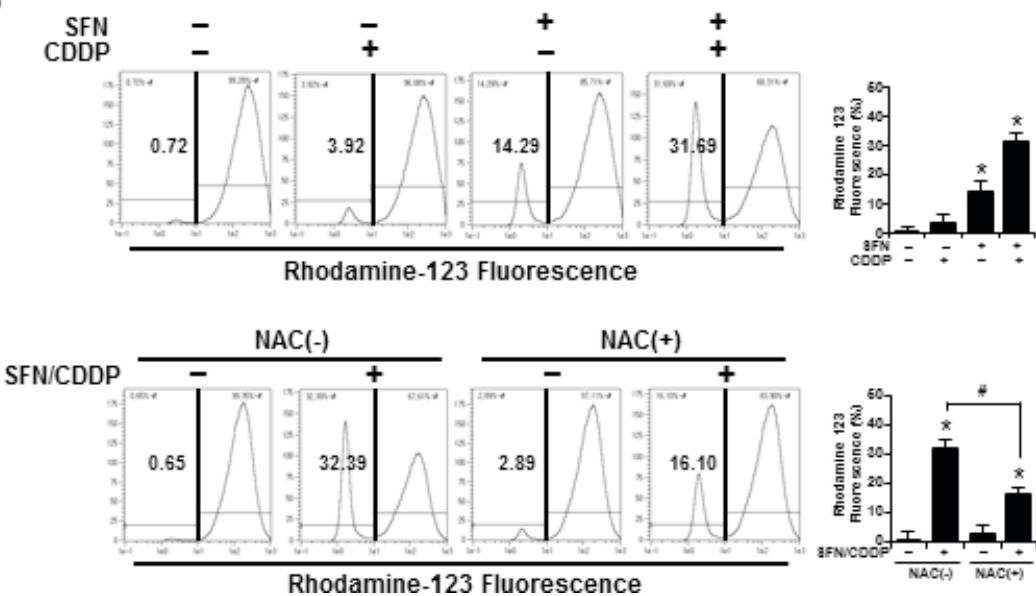

Figure 3. Effects of SFN and CDDP on ROS generation and $\triangle \Psi \mathrm{m}$ in H-28 malignant mesothelioma cells. H-28 cells were pretreated with or without NAC $(5 \mathrm{mM})$ for $2 \mathrm{~h}$ prior to treatment with SFN $(20 \mu \mathrm{M})$ and CDDP $(40 \mu \mathrm{M})$, alone or in combination, for a further $48 \mathrm{~h}$. (A) The levels of cellular ROS were measured using flow cytometry following staining with DCF-DA $(10 \mu \mathrm{M})$. A shift in DCF fluorescence to the right indicated an increase in ROS levels. (B) $\Delta \Psi \mathrm{m}$ was measured by staining the cells with Rhodamine $123(30 \mathrm{nM})$. Quantitative data are displayed as the mean \pm standard deviation for three independent experiments. ${ }^{~} \mathrm{P}<0.05$ vs. the respective untreated controls; ${ }^{~} \mathrm{P}<0.05$ vs. the respective $\mathrm{NAC}(-)$ group. $\Delta \Psi \mathrm{m}$, mitochondrial membrane potential; CDDP, cis-dichlorodiammineplatinum (cisplatin); DCF-DA, 2',7'-dichlorodihydrofluorescein diacetate; NAC, N-acetylcysteine; ROS, reactive oxygen species; SFN, sulforaphane.

The proportion of cells in the $\mathrm{G}_{2} / \mathrm{M}$ phase increased, while the percentage of cells at $\mathrm{G}_{0} / \mathrm{G}_{1}$ and $\mathrm{S}$ phases decreased, indicating $\mathrm{a}_{2} / \mathrm{M}$ phase-transition delay in the cell cycle.

To identify possible effector molecules that may be responsible for the growth-inhibiting effect of SFN/CDDP treatment, the induction of apoptosis- and cell cycle-regulating molecules was assessed by western blot analysis. As presented in Fig. 2D, SFN/CDDP induced an upregulation of p53 protein expression levels and enhanced the cleavage of procaspase- 3 and poly (ADP-ribose) polymerase (PARP), and increased the Bax/Bcl-2 expression ratio compared with cells treated with either drug alone or untreated. In addition, SFN/CDDP treatment increased the levels of $\mathrm{p}-\mathrm{Cdc} 2^{\mathrm{Ty} 15}$ and cyclin B1 protein expression levels, whereas the levels of $\mathrm{p} 21^{\mathrm{WAF1} / \mathrm{CIP} 1}$ and cyclin D1 proteins were decreased compared with the untreated control.

ROS accumulation and $\Delta \Psi m$. To determine whether the synergistic cytotoxic effects of SFN/CDDP treatment on H-28 cells were associated with oxidative mitochondrial damage, intracellular ROS levels were measured by flow cytometry using an ROS-sensitive fluorophore, DCF-DA. As presented in the representative histogram in Fig. 3A, cells treated with SFN or CDDP alone induced a slight increase in ROS accumulation, as indicated by a shift in the DCF fluorescence to the right; however, combined treatment with the two compounds increased ROS production to $38.78 \%$. In addition, the integrity of mitochondrial function was analyzed by flow cytometry using the fluorescent dye Rhodamine 123, where decreased fluorescence was correlated with a loss of $\Delta \Psi \mathrm{m}$. In the presence of SFN/CDDP, the number of cells with $\Delta \Psi \mathrm{m}$ loss increased to $31.69 \%$ (Fig. 3B). Pretreatment with the ROS scavenger NAC significantly reduced the levels of SFN/CDDP-induced ROS expression to $16.37 \%$ and lowered the number of cells with $\Delta \Psi \mathrm{m}$ loss to $16.10 \%$, compared with the NAC-untreated SFN/CDDP-induced control cells (Fig. 3A and B).

In $\mathrm{H}-28$ cells treated with SFN/CDDP, flow cytometric analysis revealed a $\mathrm{G}_{2} / \mathrm{M}$ phase-transition delay and an increase in the number of cells in the sub- $G_{0} / G_{1}$ peak, which was significantly reduced by NAC pretreatment $(\mathrm{P}<0.05$; Fig. 4A). NAC treatment also decreased the proportion 
A

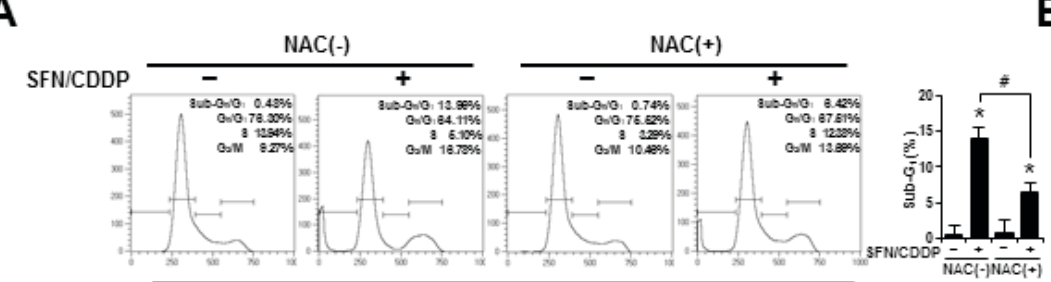

E

$\operatorname{SFN} / \operatorname{CDDP} \frac{\mathrm{NAC}(-)}{-\frac{\mathrm{NAC}(+)}{-+}}$

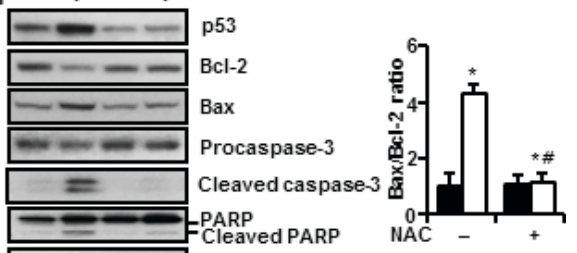

B

DNA content
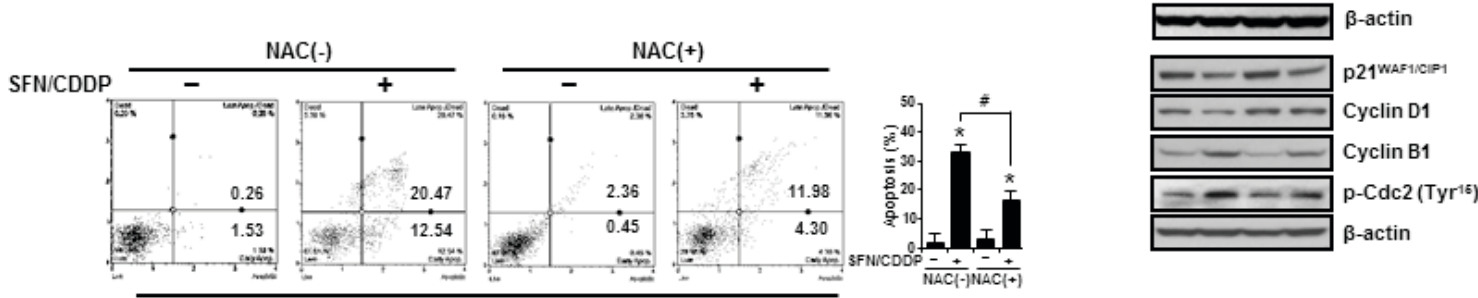

C

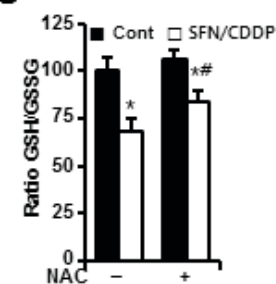

Annexin V-PE

D

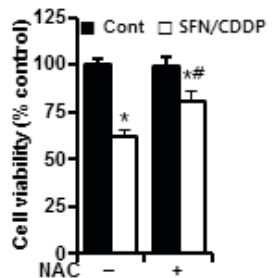

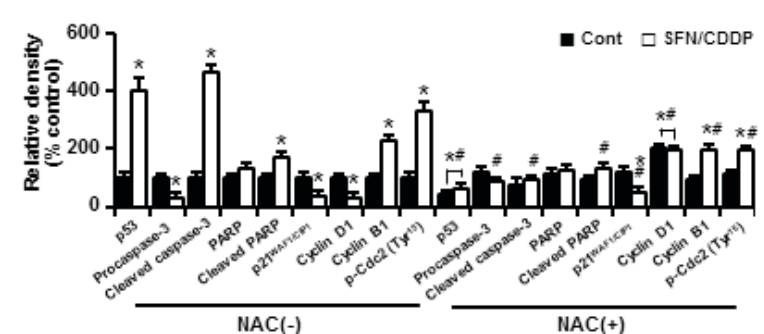

Figure 4. Protective effects of NAC against the pro-oxidant activity of SFN/CDDP in H-28 malignant mesothelioma cells. H-28 cells were pretreated with or without NAC $(5 \mathrm{mM})$ for $2 \mathrm{~h}$ prior to co-treatment with SFN (20 $\mu \mathrm{M})$ and CDDP $(40 \mu \mathrm{M})$ for a further $48 \mathrm{~h}$. (A) Cell cycle analysis was determined by propidium iodide staining. (B) Cellular apoptosis was determined by Annexin V-PE binding assay. (C) GSH/GSSG ratio was determined by GSH/GSSG-Glo assay. (D) Cell viability was determined by MTT assay. (E) Western blot analysis of apoptosis- and cell cycle-regulating proteins; $\beta$-actin was used as a loading control. The Bax/Bcl-2 ratio and relative density of protein bands were obtained from densitometric analysis of the western blot images normalized to $\beta$-actin. Error bars indicate the mean \pm standard deviation for three independent experiments. $\mathrm{P}<0.05$ vs. the respective controls; ${ }^{\#} \mathrm{P}<0.05$ vs. the respective $\mathrm{NAC}(-)$ group. Bax, Bcl-2 associated X protein; Bcl-2, B-cell lymphoma 2; CDDP, cis-dichlorodiammineplatinum (cisplatin); Cont, untreated control; GSH, reduced glutathione; GSSG, oxidized glutathione; NAC, N-acetylcysteine; PARP, poly(ADP-ribose) polymerase; p-Cdc2, phosphorylated-cyclin dependent kinase 2; PE, phycoerythrin; SFN, sulforaphane.

A
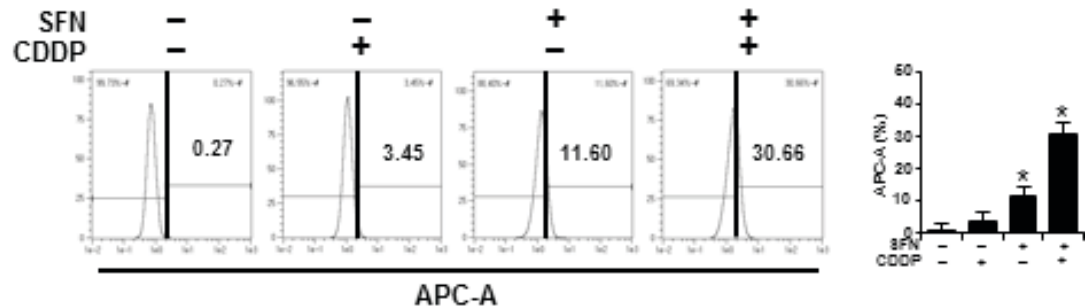

B
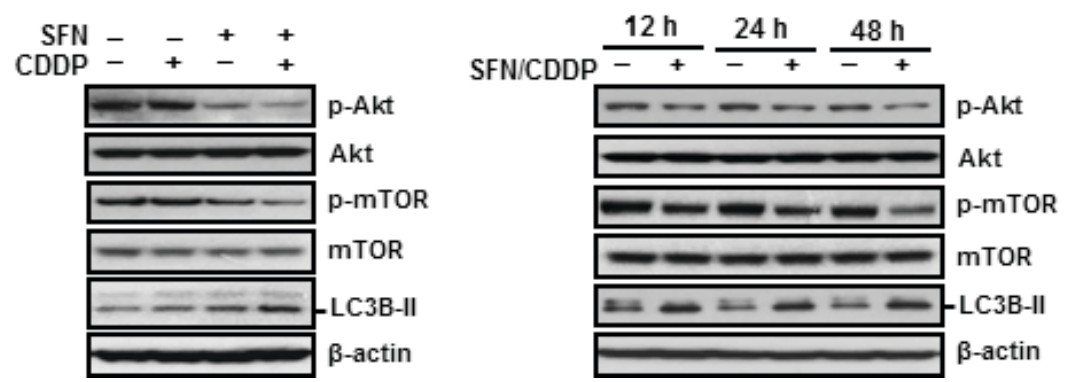

Figure 5. Autophagy-promoting effects of SFN and CDDP in H-28 malignant mesothelioma cells. (A) H-28 cells were treated with SFN (20 $\mu \mathrm{M})$ and CDDP $(40 \mu \mathrm{M})$, alone or in combination, for $48 \mathrm{~h}$. The number of LysoTracker Red(+) cells, as detected by APC-A fluorescence intensity, were analyzed by flow cytometry. (B) H-28 cells were treated with SFN $(20 \mu \mathrm{M})$ and CDDP $(40 \mu \mathrm{M})$, alone or in combination, for $48 \mathrm{~h}$ or the indicated times $(12,24$ and $48 \mathrm{~h})$, and the levels of autophagy-related proteins were measured by western blot analysis; $\beta$-actin was used as a loading control. Representative results are presented and error bars indicate the mean \pm standard deviation for three independent experiments. " $\mathrm{P}<0.05$ vs. the respective controls. APC-A, allophycocyanin channel; CDDP, cis-dichlorodiammineplatinum (cisplatin); LC3B-II, microtubule-associated protein 1 light chain 3; $\beta \mathrm{mTOR}$, mammalian target of rapamycin; p, phosphorylated; SFN, sulforaphane. 
A

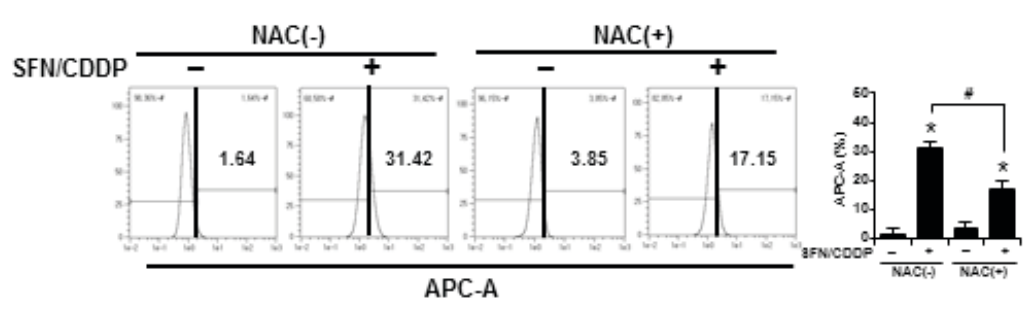

B

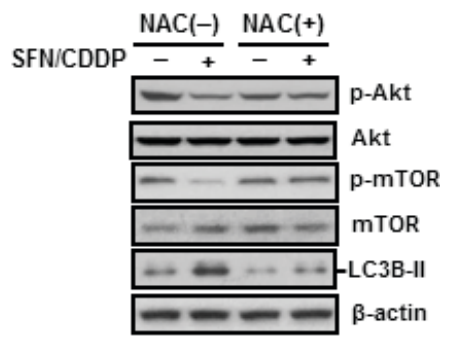

C

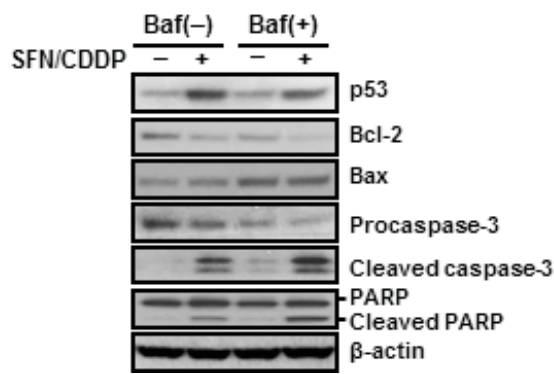

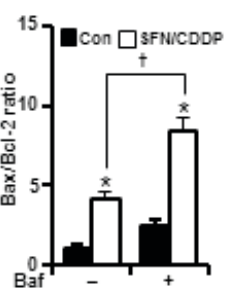

D

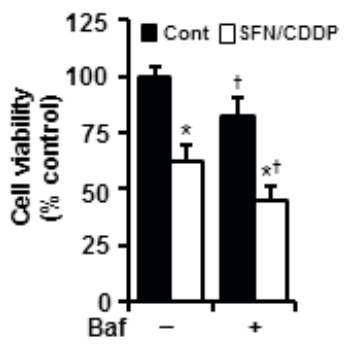

Figure 6. Effects of NAC and Baf on the SFN/CDDP-induced autophagy in H-28 malignant mesothelioma cells. (A and B) H-28 cells were pretreated with or without NAC (5 mM) for $2 \mathrm{~h}$ prior to co-treatment with SFN (20 $\mu \mathrm{M})$ and CDDP $(40 \mu \mathrm{M})$ for a further $48 \mathrm{~h}$. (A) The number of LysoTracker Red(+) cells, as detected by APC-A fluorescence intensity by flow cytometry. (B) Western blot analysis of autophagy-related proteins; $\beta$-actin was used as a loading control. (C and D) H-28 cells were pretreated with or without Baf (50 nM) for $2 \mathrm{~h}$ prior to co-treatment with SFN (20 $\mu \mathrm{M})$ and CDDP (40 $\mu \mathrm{M})$ for a further 48 h. (C) Western blot analysis of apoptosis-regulating proteins; $\beta$-actin was used as a loading control. (D) Cell viability was determined by MTT assay. Representative results are presented and error bars indicate the mean \pm standard deviation for three independent experiments. ${ }^{*}<0.05$ vs. the respective controls; ${ }^{\#} \mathrm{P}<0.05$ vs. the respective NAC(-) group; ${ }^{\mathrm{P}}<0.05$ vs. the respective Baf(-) group. Baf, bafilomycin A1; Bax, Bcl-2 associated $\mathrm{X}$ protein; Bcl-2, B-cell lymphoma 2; CDDP, cis-dichlorodiammineplatinum (cisplatin); Cont, untreated control; LC3B-II, microtubule-associated protein 1 light chain $3 \beta$; mTOR, mammalian target of rapamycin; NAC, N-acetylcysteine; p, phosphorylated; PARP, poly(ADP-ribose) polymerase; SFN, sulforaphane.

Annexin V-PE-positive cells ( $\mathrm{P}<0.05$; Fig. 4A and B). This was accompanied by a significant improvement in the GSH/GSSG ratio $(\mathrm{P}<0.05)$, and cell viability increased to 85.53 and $81 \%$ in the SFN/CDDP-treated cells respectively, compared with the NAC-untreated SFN/CDDP-induced control cells (68.6 and $61.9 \%$, respectively) $(\mathrm{P}<0.05$; Fig. 4C and D). Furthermore, cells exposed to SFN/CDDP and pretreated with NAC exhibited a decrease in the expression level of p53 protein, an increase in the level of cyclin D1 protein, a decrease in the Bax/Bcl-2 ratio, a reduction in the resultant cleavages of procaspase- 3 and PARP, and a slight decrease in the phosphorylation of Cdc 2 on Tyr $^{15}$, compared with SFN/CDDP-treated cells that were not exposed to NAC (Fig. 4E). However, there were no significant differences in the expression levels of $\mathrm{p} 21^{\mathrm{WAF} 1 / \mathrm{CIP} 1}$ and cyclin $\mathrm{B} 1$ proteins between the two treatment groups.

ROS-dependent activation of autophagy. To assess the autophagy-inducing effects of SFN and CDDP treatment on $\mathrm{H}-28$ cells, cells were treated for $48 \mathrm{~h}$ with SFN and CDDP, alone or in combination, and the acidic vacuoles were subsequently stained with LysoTracker Red DND-99 and analyzed on channel APC-A by flow cytometry. As presented in Fig. 5A, treatment with SFN/CDDP or SFN alone exhibited a significant increase in cells displaying high LysoTracker Red fluorescence, indicative of autophagy. Compared with either SFN or CDDP treatments alone, treatment with SFN/CDDP resulted in higher protein expression levels of the autophagy marker LC3B-II and a decrease in the phosphorylation of Akt and $\mathrm{mTOR}$, and these alterations were detected at after 12 , 24 and $48 \mathrm{~h}$ treatment (Fig. 5B). These expression patterns were reversed by NAC pretreatment, suggesting the involvement of ROS in the activation of autophagy in response to SFN/CDDP exposure (Fig. 6A and B). Pretreatment with the autophagy inhibitor bafilomycin A1, which prevents autophagosome-lysosome fusion, enhanced the expression levels of cleaved caspase- 3 and cleaved PARP, led to a further increase in the Bax/Bcl-2 ratio (Fig. 6C), and significantly enhanced SFN/CDDP-induced cytotoxicity (Fig. 6D).

\section{Discussion}

The present study investigated the effects of SFN co-treatment on enhancing the anticancer role, along with the phenomena of apoptosis and autophagy in this process, of CDDP in H-28 malignant mesothelioma cells, and identified alterations in apoptotic and autophagic factors. First, SFN/CDDP treatment produced a significant and synergistic cytotoxic effect, evidenced by the enhancement of apoptosis and $\mathrm{G}_{2} / \mathrm{M}$ phase-transition delay in the cell cycle compared with either treatment alone. Second, this synergism appeared to be mediated via proapoptotic and cell-cycle modulators, including p53, Bcl-2, Bax, phosphatidylinositol-3-kinase (PI3K)/Akt and Cdc2. Third, the pro-oxidant activity of SFN/CDDP treatment contributed to the simultaneous induction of apoptosis and autophagy. Fourth, inhibition of autophagy by bafilomycin A1 augmented SFN/CDDP-induced cytotoxicity, indicating SFN/CDDP-induced autophagy is a pro-survival process in H-28 cells.

Cells are continually receiving and integrating a variety of positive and negative growth signals. Loss of normal survival 
signals or an increase in negative growth signals tips the balance and a cell may undergo programmed cell death (15). Cancer cells may be able to survive and thrive against cellular stresses by weakening or crippling the proapoptotic machinery, for example via p53 inactivation and upregulation of antiapoptotic Bcl-2-related proteins (16). In this regard, the presence of upregulated p53 expression, increased $\mathrm{Bax} / \mathrm{Bcl}-2$ ratio and inhibited pro-survival PI3K/Akt signaling, which suppresses the actions of the proapoptotic circuitry, may provide a theoretical basis for the production of a synergistic apoptosis-promoting effect by SFN/CDDP treatment in $\mathrm{H}-28$ cells. In addition, the concurrence of $\mathrm{G}_{2} / \mathrm{M}$ phase-transition delay with apoptosis observed in the present study is consistent with a previous report that revealed reduced viability in ovarian cancer cells treated with SFN in triple combination with epigallocatechin gallate and CDDP (17). However, that observation differs from the results of the present discovery, in which the increase in cells at the $\mathrm{G}_{2} / \mathrm{M}$ phase (indicative of an arrest) was not dependent on the cyclin dependent kinase inhibitor $\mathrm{p} 21^{\mathrm{WAF} 1 / \mathrm{CIP} 1}$, rather it was associated with an upregulation of $\mathrm{p}-\mathrm{Cdc} 2$ expression levels. The phosphorylation of $\mathrm{Cdc} 2$ on $\mathrm{Tyr}^{15}$ has been reported to render the $\mathrm{Cdc} 2 /$ cyclin $\mathrm{B}$ complex inactive, thus keeping the cells in $\mathrm{G}_{2}$ phase from entering $\mathrm{M}$ phase (18).

Oxidative stress-induced damage to macromolecules has been proposed as a possible mechanism for CDDP-induced cytotoxicity (19). Previous research has also demonstrated that SFN treatment increases the sensitivity of various cancer cells to chemotherapeutic agents, by inducing a further increase in oxidative stress $(8,9)$. Excessive ROS production triggers mitochondrial-mediated apoptosis by increasing the $\mathrm{Bax} / \mathrm{Bcl}-2$ ratio, which results in marked $\Delta \Psi$ m loss and subsequent caspase activation (20). Combining these results with observations from the present study, which revealed mitochondrial damage and an increase in apoptosis, indicated that the pro-oxidant role of SFN is essential in potentiating the cytotoxic effects of CDDP. NAC is a precursor for cellular biosynthesis of the antioxidant GSH and stimulates the synthesis of GSH. Exposure to SFN/CDDP resulted in a significant reduction in the GSH/GSSG ratio in H-28 cells; however, this was reversed in the presence of NAC. The replenishment of cellular GSH levels may explain, at least in part, the effect of NAC on reducing ROS levels. The concurrence of autophagy with apoptosis in response to SFN/CDDP exposure was apparently dependent on ROS accumulation. Consistent with our data, previous studies indicated that ROS may act as upstream molecules in mediating autophagy and apoptosis $(21,22)$. Furthermore, elevated ROS levels suppress the PI3K/Akt/mTOR survival pathway and subsequently induce apoptosis and autophagy, whereas activation of this survival pathway inhibits several autophagy-promoting proteins via phosphorylation of mTOR complex $1(23,24)$. This rationale is supported by the present study which demonstrated that SFN/CDDP treatment reduced the phosphorylation of both Akt and mTOR, induced the upregulation of LC3B-II expression and caused a significant increase in the proportion of LysoTracker Red(+) cells. $\mathrm{PI} 3 \mathrm{~K} / \mathrm{Akt} / \mathrm{mTOR}$ signaling is a crucial survival pathway in MM (25). Recently, Echeverry et al (26) reported that autophagy is one of the predominant mechanisms of drug resistance to the dual PI3K/mTOR inhibitors, NVP-BEZ235 and GDC-0980, in MM cells. Bcl-2 has also been reported as a possible effector molecule linked to both apoptosis and autophagy, as it inhibits apoptosis by preventing mitochondrial cytochrome $c$ release and suppresses autophagy by interacting with Beclin-1 (27). In the present study, the downregulation of Bcl-2 protein expression in $\mathrm{H}-28$ cells exposed to SFN/CDDP may have contributed to the concurrence of apoptosis and autophagy; however, further research is required to understand the significance of this double role of Bcl-2.

A number of previous studies have reported on the paradoxical functions of autophagy in cancer, in which it can either promote or inhibit cell survival (11-13). Cancer cells may take advantage of this mechanism for protection and to maintain survival against stresses. Evidence of autophagy as a survival mechanism comes from previous research that indicated that the activation of autophagy contributes to cancer progression, therapeutic resistance and survival of dormant cancer cells $(12,28)$. The present study demonstrated that autophagic inhibition potentiated the cytotoxic effect of SFN/CDDP treatment, which supports the cytoprotective role of autophagy. However, significant levels of apoptotic cell death were observed, and the activation of autophagy was not able to override $\mathrm{H}-28$ apoptosis induced by SFN/CDDP treatment. Detailed mechanistic studies under the same conditions are required to unveil the cytoprotective aspects of autophagy.

In conclusion, SFN/CDDP treatment produced a synergistic outcome in H-28 cells via activation of the ROS-dependent mitochondrial apoptotic pathway as a fail-safe mechanism designed to eliminate damaged cells from the growing population. Autophagy was additionally activated as a prosurvival process to SFN/CDDP insults, possibly as an adaptive survival mechanism. These responses may contribute to the selection of more malignant clones that escape apoptotic eradication, and may therefore provide a route for drug-resistant subpopulations to arise. From this perspective, the present study underlines the concept that targeting autophagic regulation may be an attractive therapeutic strategy for the improvement of clinical outcomes for patients with MM.

\section{Acknowledgements}

This research was supported by the Basic Science Research Program through the National Research Foundation of Korea (NRF), funded by the Ministry of Education (grant no. NRF-2015R1D1A3A03020269).

\section{References}

1. Wolff H, Vehmas T, Oksa P, Rantanen J and Vainio H: Asbestos, asbestosis, and cancer, the helsinki criteria for diagnosis and attribution 2014: Recommendations. Scand J Work Environ Health 41: 5-15, 2015.

2. Raptopoulos V: Peritoneal mesothelioma. Crit Rev Diagn Imaging 24: 293-328, 1985.

3. Scripcariu V, Dajbog E, Radu I, Ferariu D, Pricop A, Grigoraş M and Dragomir C: Malignant peritoneal mesothelioma tumours. Evolution, treatment, prognosis: Rev Med Chir Soc Med Nat Iasi 111: 673-677, 2007.

4. Ray M and Kindler HL: Malignant pleural mesothelioma: An update on biomarkers and treatment. Chest 136: 888-896, 2009.

5. Villanova F, Procopio A and Rippo MR: Malignant mesothelioma resistance to apoptosis: Recent discoveries and their implication for effective therapeutic strategies: Curr Med Chem 15: 631-641, 2008. 
6. Vogelzang NJ, Rusthoven JJ, Symanowski J, Denham C, Kaukel E, Ruffie P, Gatzemeier U, Boyer M, Emri S, Manegold C, et al: Phase III study of pemetrexed in combination with cisplatin versus cisplatin alone in patients with malignant pleural mesothelioma. J Clin Oncol 21: 2636-2644, 2003.

7. Tomczyk $\mathbf{J}$ and Olejnik A: Sulforaphane-a possible agent in prevention and therapy of cancer. Postepy Hig Med Dosw (Online) 64: 590-603, 2010 (In Polish).

8. Lin LC, Yeh CT, Kuo CC, Lee CM, Yen GC, Wang LS, Wu CH, Yang WC and Wu AT: Sulforaphane potentiates the efficacy of imatinib against chronic leukemia cancer stem cells through enhanced abrogation of Wnt/ $\beta$-catenin function. J Agric Food Chem 60: 7031-7039, 2012.

9. Kim H, Kim EH, Eom YW, Kim WH, Kwon TK, Lee SJ and Choi KS: Sulforaphane sensitizes tumor necrosis factor-related apoptosis-inducing ligand (TRAIL)-resistant hepatoma cells to TRAIL-induced apoptosis through reactive oxygen species-mediated up-regulation of DR5. Cancer Res 66: 1740-1750, 2006.

10. Sharma K, Le N, Alotaibi M and Gewirtz DA: Cytotoxic autophagy in cancer therapy. Int J Mol Sci 15: 10034-10051, 2014

11. Giuliani CM and Dass CR: Metabolic stress and cancer: Is autophagy the common denominator and a feasible target? J Pharm Pharmacol 66: 597-614, 2014.

12. Vlahopoulos S, Critselis E, Voutsas IF, Perez SA, Moschovi M, Baxevanis CN and Chrousos GP: New use for old drugs? Prospective targets of chloroquines in cancer therapy. Curr Drug Targets 15: 843-851, 2014.

13. Jin S and White E: Role of autophagy in cancer: Management of metabolic stress. Autophagy 3: 28-31, 2007.

14. Chou TC and Talalay P: Quantitative analysis of dose-effect relationships: The combined effects of multiple drugs or enzyme inhibitors. Adv Enzyme Regul 22: 27-55, 1984.

15. Mehlen P and Thibert C: Dependence receptors: Between life and death. Cell Mol Life Sci 61: 1854-1866, 2004.

16. Siddik ZH: Cisplatin: Mode of cytotoxic action and molecular basis of resistance. Oncogene 22: 7265-7279, 2003.

17. Chen H, Landen CN, Li Y, Alvarez RD and Tollefsbol TO: Enhancement of cisplatin-mediated apoptosis in ovarian cancer cells through potentiating $\mathrm{G} 2 / \mathrm{M}$ arrest and p21 upregulation by combinatorial epigallocatechin gallate and sulforaphane. J Oncol 2013: 872957, 2013.
18. Singh SV, Herman-Antosiewicz A, Singh AV, Lew KL, Srivastava SK, Kamath R, Brown KD, Zhang L and Baskaran R: Sulforaphane-induced G2/M phase cell cycle arrest involves checkpoint kinase 2-mediated phosphorylation of cell division cycle 25C. J Biol Chem 279: 25813-25822, 2004.

19. Deavall DG, Martin EA, Horner JM and Roberts R: Drug-induced oxidative stress and toxicity. J Toxicol 2012: 645460, 2012.

20. Deng S, Tang S, Zhang S, Zhang C, Wang C, Zhou Y, Dai C and $\mathrm{Xiao} \mathrm{X}$ : Furazolidone induces apoptosis through activating reactive oxygen species-dependent mitochondrial signaling pathway and suppressing PI3K/Akt signaling pathway in HepG2 cells. Food Chem Toxicol 75: 173-186, 2015.

21. Dunlop EA and Tee AR: mTOR and autophagy: A dynamic relationship governed by nutrients and energy. Semin Cell Dev Biol 36: 121-129, 2014.

22. Nazio F and Cecconi F: mTOR, AMBRA1, and autophagy: An intricate relationship. Cell Cycle 12: 2524-2525, 2013.

23. Chen B, Li D, Li M, Li S, Peng K, Shi X, Zhou L, Zhang P, $\mathrm{Xu} \mathrm{Z}$, Yin $\mathrm{H}$, et al: Induction of mitochondria-mediated apoptosis and PI3K/Akt/ mTOR-mediated autophagy by aflatoxin B2 in hepatocytes of broilers. Oncotarget 7: 84989-84998, 2016.

24. Mi S, Xiang G, Yuwen D, Gao J, Guo W, Wu X, Wu X, Sun Y, $\mathrm{Su}$ Y, Shen Y and Xu Q: Inhibition of autophagy by andrographolide resensitizes cisplatin-resistant non-small cell lung carcinoma cells via activation of the Akt/mTOR pathway. Toxicol Appl Pharmacol 310: 78-86, 2016.

25. Zhou S, Liu L, Li H, Eilers G, Kuang Y, Shi S, Yan Z, Li X, Corson JM, Meng F, et al: Multipoint targeting of the PI3K/mTOR pathway in mesothelioma. Br J Cancer 110: 2479-2488, 2014

26. Echeverry N, Ziltener G, Barbone D, Weder W, Stahel PA, Broaddus VC and Felley-Bosco E: Inhibition of autophagy sensitizes malignant pleural mesothelioma cells to dual PI3K/mTOR inhibitors. Cell Death Dis 6: e1757, 2015.

27. Zhao GX, Pan H, Ouyang DY and He XH: The critical molecular interconnections in regulating apoptosis and autophagy. Ann Med 47: 305-315, 2015.

28. Lu Z, Luo RZ, Lu Y, Zhang X, Yu Q, Khare S, Kondo S, Kondo Y, $\mathrm{Yu}$ Y, Mills GB, et al: The tumor suppressor gene ARHI regulates autophagy and tumor dormancy in human ovarian cancer cells. J Clin Invest 118: 3917-3929, 2008. 